\title{
Phänomenologische Probleme des Sehens
}

\author{
JESÚS PADILLA GÁLVEZ
}

\section{Einführung}

Es ist Edmund Husserl zu verdanken, dass er in Zusammenhang mit der Grammatik auf das Problem der selbstständigen und unselbstständigen Bedeutung hingewiesen hat. Innerhalb des Kapitels 'Der Unterschied der selbstständigen und unselbstständigen Bedeutungen und die Idee der reinen Grammatik' seines Werkes 'Logische Untersuchungen' macht er auf einen Unterschied bei der Untersuchung der Meinongschen Objekte ${ }^{1}$ aufmerksam, indem er, wie im Beispiel des runden Vierecks, zwischen Unsinn und Widersinn ${ }^{2}$ unterscheidet. Er besteht also darauf, das Unsinnige oder Sinnlose vom Widersinnigen und Absurden $\mathrm{zu}$ trennen. Der Meinongschen Verknüpfung "rundes Viereck" spricht er zwar die Existenz eines entsprechenden Objekts ab, gesteht ihm aber eine ideale Bedeutung zu. Um solche Verknüpfungen genauer zu untersuchen muss man vorher einige die Grammatik betreffende Fragen beantworten. Ihre Lösung besteht darin, dass die Vereinheitlichung der Bedeutungen von "rund" und "Viereck" keinem Gegenstand entspricht. Die Begriffe "rund" und von "Viereck" sind sowohl im Bereich der Vorstellung als auch in dem der Gegenstände miteinander unverträglich. E. Husserl bleibt dem Bereich der Gegenständlichkeit verhaftet, wenn er zwar die Bedeutung von "rundes Viereck" als existierend anerkennt, aber ihr gleichzeitig keinen existierenden Gegenstand zuordnen kann.

In diesem Zusammenhang erstaunt es, dass L. Wittgenstein am Anfang eines Absatzes mit dem Titel 'Das sehende Subjekt und der Gesichtsraum' in 'The Big Typescript' folgendes behauptet:

${ }^{1}$ Meinong 1978, 481-530.

${ }^{2}$ Husserl $L U, \mathrm{II} / 1,1968,326 \mathrm{ff}$.

Phenomenology as Grammar (Ed. Jesús Padilla Gálvez), 141-158.

(C) Ontos Verlag, Frankfurt a. M. 
"Es ist unsinnig zu sagen "ich sehe die Dinge / diesen Gegenstand / im Gesichtsraum".,"

Wenn man diesen ersten Satz liest, so erscheint L. Wittgensteins Behauptung zunächst so, als ob der prototypische Satz aus der Wahrnehmungstheorie unsinnig wäre. ${ }^{4}$ Normalerweise fragt man sich dann nach dem Grund für die Unsinnigkeit und wieso es unsinnig sei, alle Objekte der Umgebung zu sehen, die das ruhende Auge wahrnimmt. Aber diese erste Lektüre hat die Anführungszeichen nicht in Betracht gezogen und bei näherer Betrachtung rückt "Gesichtraum" in den Vordergrund der Untersuchung. Bei eingehender Lektüre merkt man, dass L. Wittgenstein einen in Anführungszeichen stehenden Satz als Unsinn bezeichnet und jeden Sinn abspricht. Es stellt sich folgende Frage: Geht es hier also um die Unsinnigkeit bestimmter Formulierungen, die das sehende Subjekt über den Gesichtsraum ausdrückt? E. Husserl sagt diesbezüglich, dass der Satz "Ich sehe die Dinge / diesen Gegenstand / im Gesichtsraum" zwar wahrhaft eine einheitliche Bedeutung liefert, die "... ihre Weise der "Existenz", des Seins in der "Welt" der idealen Bedeutungen hat; aber es ist eine apodiktische Evidenz, daß der existierenden Bedeutung kein existierender Gegenstand entsprechen kann., ${ }^{5}$ Leider ist für den von L. Wittgenstein vorgeschlagenen Satz die von E. Husserl vorgeschlagene Antwort nicht schlüssig, da es in der semantischen Problemstellung der Bedeutung nicht um die Frage der Existenz des Seins in der Welt geht, sondern um den passenden Ausdruck, den man benutzen soll um den Zusammenhang zwischen Dingen bzw. Gegenständen und Gesichtraum zu analysieren. Das veranlasst Wittgenstein zu folgender Frage:

"Im Gegensatz wozu? Ist es denkbar, daß ich sie / ihn / höre, oder daß ein Anderer sie / ihn / sieht?"6

Bekämen wir im Rahmen einer Prüfung im Fach Physiologie eine Frage gestellt oder würden wir uns in der Kunstakademie befinden und gefragt werden, ob wir Gegenstände sehen oder hören, so würde sicherlich jeder diese Fragen bejahen. Diese Antwort lässt sich dadurch erklären, dass wir die Hypothese des Sehens von Objekten aufgrund der Erfahrung immer rechtfertigen können. Die seit der Neuzeit vorgeschlagene Vorgangsweise

\footnotetext{
${ }^{3}$ Wittgenstein $B T, 2000,310$.

${ }^{4}$ Siehe z.B. die Beschreibung E. Machs über sein Gesichtsfeld in: Mach 1922 (1991), $15 f$.

${ }^{5}$ Husserl $L U$, II/1, 1968, 326.

${ }^{6}$ Wittgenstein $B T, 2000,310$.
} 
haben wir gelernt und wir gehen fest davon aus, sie bei jeder Beobachtung anwenden zu können. Wenn wir beispielweise eine Rose im Garten wahrnehmen, so greifen wir auf unsere Erfahrung mit dem Gegenstand den wir "Rose" nennen zurück. Danach stellen wir eine Hypothese auf der Grundlage der oben angesprochenen Beobachtung auf. Und erst in einem nächsten Schritt können wir eine Regel aufstellen, die die Form einer Art Allhypothese annimmt. Diese Vorgangsweise hilft uns, den Aufbau des Satzes im ersten Zitat zu verstehen, wo behauptet wird, dass ein sehendes Subjekt die Dinge bzw. einen Gegenstand im Gesichtsraum wahrnimmt. Das Rätsel hat sich damit gelöst, obwohl man fragen könnte, ob es das eigentliche Rätsel war? Oder anders gesagt: hat diese Fragestellung überhaupt allgemeingültigen Charakter? Fragen wir ein Kind, wieso es keine Tomaten isst oder ob ihm eine Gurke schmecke, so antwortet es vielleicht dass es das rote Ding nicht möge oder ihm der grüne Gegenstand nicht schmecke. Kann es aber das wahrnehmbare Rot schmecken oder kann das Grün der Gurke den Geschmack beeinflussen? Oder bildet das Kind einfach unsinnige Sätze? ${ }^{7}$ In diesem Fall würde Edmund Husserl auf die "normale Funktion" hinweisen, die dem Ausdruck seine Bedeutung verleiht. ${ }^{8}$ Als Grund für die Unsinnigkeit verwiese er dann auf die Unverträglichkeit zwischen "Ding" und "Gesichtraum". Diese These bleibt der Ebene der Gegenständlichkeit verhaftet, liefert aber keine Erklärung dafür, wieso ein Gegenstand unverträgliche Bedeutungen haben kann. Sie stellt nur die Existenz der Dinge als Kriterium für die Unsinnigkeit des "roten Geschmacks" in den Vordergrund.

Ist L. Wittgenstein auf dem richtigen Weg, wenn er die Theorie des hypothetischen Räsonierens mittels irrealer Konditionalsätze hinterfragt? Seine Fragen sind dazu geeignet das Problem von einer anderen Seite zu beleuchten. Er stellt an das Subjekt, das sich mit dem Hervorbringen eines wissenschaftlichen Satzes begnügt, die Frage, nach welcher Regel es beim

\footnotetext{
${ }^{7}$ Man braucht kein Kind zu sein, um solche unsinnigen Paare zu bilden. Thomas Mann bekam den Nobelpreis für den "Zauberberg", obwohl es keinen Sinn macht einen Berg als bezaubert zu bezeichnen. Edmund Husserl bevorzugt die Beispiele des runden Vierecks, des hölzernen Eisens oder alle Vierecke haben fünf Ecken, um unsinnige (d.h. sinnlose) grammatikalische Ausdrücke zu untersuchen. Husserl LU, II/1, 1968, 327.

${ }^{8}$ Husserl $L U, \mathrm{II} / 1,1968,326 \mathrm{f}$.

${ }^{9}$ Husserl LU, II/1, 1968, 327.
} 
Sehen vorgeht. Erstaunlicherweise kann diese Frage mit dem Verständnis der neuzeitlichen Methode nicht zufriedenstellend beantwortet werden. Sowohl die Frage, als auch die daraus resultierende Antwort werden für unsinnig gehalten, da sie keine naturwissenschaftlichen Sätze sind. Wie bereits oben erwähnt, werden naturwissenschaftliche Sätze auf der Grundlage von Beobachtungen aufgestellt und scheinen eine Erklärung für das Sehen abzugeben. Man könnte aber unmittelbar fragen, ob die naturwissenschaftlichen Sätze das leisten. Wie in der neuzeitlichen Wissenschaft stehen uns Hypothesen zur Verfügung: Man hat sich bisher eher um Antworten auf Fragen gekümmert, wie etwa, wieso die Blume namens "Rosa" hier und jetzt ein Gegenstand ist. Man hat sich dabei eher der Frage zugewandt, wieso die Rose im Garten, also im Raum, wahrgenommen wird. Ähnliches gilt für die Phänomenologie Husserls: indem er solche Verknüpfungen wie "rundes Viereck" hinterfragt, drängt sich die Frage in den Vordergrund, ob ein "Gegenstand", der gleichzeitig "rund" und "viereckig" wäre, überhaupt existiere? Dieser Gegenstand "rundes Viereck" kann gar nicht existieren, da sich zwar jeder einen runden und einen viereckigen Gegenstand vorstellen kann, aber beide Formen zusammen genommen eine "sinnlose" Figur darstellen und daher beide Wörter miteinander unverträglich sind. Daher muss die Phänomenologie die Verknüpfung "rundes Viereck" wegen der Unverträglichkeit eines gleichzeitig runden und viereckigen Gegenstands ablehnen. Kann diese Methode der Unverträglichkeit auch auf den Satz "ich sehe die Dinge / diesen Gegenstand / im Gesichtsraum" angewendet werden? Ist eine naturwissenschaftliche Aussage wirklich eine Erklärung dafür, was wir sehen? Sind also am Ende die von L. Wittgenstein gestellten Fragen unsinnig? Um all diesen Fragen eine Antwort zuzuführen müssen wir uns mit der hypothetischen Methode auseinandersetzen.

\section{Die hypothetische Methode}

Das Problem sei nochmals mittels eines einfachen Beispiels dargestellt. Wenn jemand sagt:

(1) Die Rose ist eine rote Blume,

so kann dieser Satz vielerlei bedeuten. Es kann heissen, dass wir eine Zierflanze mit duftenden Blüten und dornigem Stiel sehen. Wir könnten auch sagen, dass die Farbe der Zierpflanze auf einer Wellenlänge zwischen 600 
$\mathrm{mm}$ und $780 \mathrm{~mm}$ liegt. Oder auch, dass mir jemand eine Blume schenkte, die eine politisch links stehende Partei als Symbol benutzt. Alle diese Beschreibungen versuchen "Rose" aufgrund ihrer Farbe zu bestimmen. ${ }^{10}$ Ein Hörer kann in verschiedenen Kontexten zumindest verstehen, was ein Sprecher sagen möchte. Von einem Hörer außerhalb unseres Kulturkreises, wie etwa dem Zugehörigen eines Amazonasvolkes, mit dem wir keine gemeinsame Erfahrung haben, würden wir mit solch einer Definition von "Rose" möglicherweise nur Unverständnis ernten. Wenn wir also sagen:

(2) Letztes Jahr schenkte mir jemand rote Rosen,

so könnte der Sprecher von (2) mit dem Ausdruck "rote Rosen" gewöhnlich folgende Bedeutung ausdrücken: Ich glaube, dass mir jemand letztes Jahr zu irgendeinem Ereignis Rosen schenkte, da ich etwas Rotes gesehen habe, das so aussah und so roch und in unserer Kultur ein Symbol für etwas ist. Wenn man mich nach Rosen fragt, so wird von mir erwartet, dass ich alle diese Beschreibungen aufzähle. Die Bestimmung von denjenigen "Rosen", die ich letztes Jahr geschenkt bekam, wäre also: die Blumen, auf die alles oben genannte zutrifft, können als Rosen identifiziert werden.

Wenn sich aber nun Einiges davon als falsch erwiese, weil ich etwa nur phantasiert oder eine Geschichte erfunden hätte und gar keine Rosen geschenkt bekommen, sondern selbst gekauft hätte, wäre dann der Satz (2) als falsch anzusehen? Der Satz (2) wäre nicht falsch, wenn dieser "Jemand" durch "mich selbst" ersetzt werden könnte. Dann hieße der Satz: "Letztes Jahr schenkte ich mir rote Rosen." Zwar wäre der Satz etwas paradox, würde man aber den Begriff "schenken" anstelle von "kaufen" gebraucht. Nehmen wir an, der heimliche Verehrer, der keine Rosen schenkte, heiratete die Verehrte totzdem. Würde dann die Heiratsabsicht falsch sein? Wenn es dem Verehrer, ohne dass er seine Verehrung zum Ausdruck gebracht hat, gelungen wäre die Partnerin zu heiraten, wäre der ursprüngliche Satz dann falsch? Wo beginnt und wo endet die hauptsächliche Bedeutung? Man könnte sogar so weit gehen und sagen, dass wir den Ausdruck "Rosen" in gewissem Sinne ohne feste Bedeutung gebrauchen, oder dass Sprecher und Hörer bereit sind, die Bedeutungen des Ausdrucks je nach veränderten Spielregeln zu verstehen.

${ }^{10}$ Siehe: Russell 1956, 41-56. 
Wenn wir vor einer optischen Prüfung stehen und gefragt werden, was wir unter "Rose" verstehen, so würde von uns erwartet, dass wir gemäß der bereits erläuterten Vorgehensweise antworten, die Blume habe z.B. im Falle einer roten Rose eine Wellenlänge zwischen 600 und $780 \mathrm{~mm}$ und es lägen Anzeichen vor, die auf eine Rose hindeuteten. Ist dieser Satz aber eine Antwort auf die ursprüngliche Frage? Selbstverständlich ist es eine Antwort auf meine Frage und in einem bestimmten Kontext ist es die richtige Antwort, die vom Sprecher erwartet wird. Aber verstehen wir unter "rote Rose" genau die der Farbe entsprechende Wellenlänge, bzw. wird mit der Wellenlänge einer bestimmten Blumenfarbe die Frage eindeutig beantwortet? Im Prinzip könnte ein Prüfer den Satz als korrekt ansehen, weil er auf der Grundlage einer Hypothese aufgebaut wurde. Die in der Neuzeit übliche Vorgangsweise basiert auf einer Wahrnehmung, wie z. B. dem Sehen der Farbe Rot, die einer bestimmten Wellenlänge entspricht. Auf der Grundlage dieser Beobachtung wird dann eine Hypothese aufgestellt. Als Resultat steht am Ende eine Reihe von naturwissenschaftlichen Sätzen. Dieses Verfahren geht von der Annahme aus, dass ein sehendes Subjekt die Dinge bzw. einen Gegenstand im Gesichtsraum sieht, dem bestimmte Eigenschaften entsprechen. In der neuzeitlichen Wissenschaft wird das sehende Subjekt durch ein Modell ersetzt das erlaubt, die Farbe des Gegenstandes wahrzunehmen und zu messen. Bei der Messung geht es um die Ersetzung der rein qualitativen Eigenschaften des Dings durch quantitative, damit "rot" in einer Skala immer eindeutig bestimmt werden kann. Immer wenn diese Skala eine Wellenlänge von $600 \mathrm{~mm}$ bis $780 \mathrm{~mm}$ anzeigt, können wir die Farbe der Blume als "rot" kennzeichnen und dann als rote Rose bestimmen. Darüberhinaus steht aber zur Diskussion, warum wir einen Gegenstand wahrnehmen und welche Erklärung wir für diese Wahrnehmung geben können. Oben habe ich eine quantitative Skala herangezogen, die mir die Farbe des Gegenstandes bestätigt.

Als wir nach einer Erklärung für (1) suchten, erhielten wir eine Reihe von Beschreibungen, von denen einige als Definitionen fungieren konnten. Auf die Frage "Was verstehen Sie unter "rote Rose"? erhielten wir eine Reihe von Antworten, die nicht gänzlich regellos waren. Trotz genauerer Untersuchung haben wir keine konkretere Bestimmung erhalten als das, was 
L. Wittgenstein ein "Schwanken zwischen mehreren Bedeutungen"" nennt. Wenn gefragt wird was wir eigentlich mit "Rose" genau meinen, wird es schwieriger. Dabei sind vor allem zwei Elemente hervorzuheben, erstens, dass man Definitionen mit der Absicht vorgibt, das zuerst gegebene Zeichen durch ein neues zu ersetzen. ${ }^{12}$ Zweitens, dass bei diesen Ersetzungen auch undefinierte Elemente vorkommen, die als verschwommene Ränder fungieren.

Aber kehren wir nochmals zu den von L. Wittgenstein aufgestellten Fragen zurück. Haben wir die Bedeutung von "Rose" bestimmt? Ich stelle meine Tochter vor unseren Rosengarten und bitte sie, die Zierpflanze mit duftenden Blüten und dornigem Stiel mit der Farbe des Blutes abzuschneiden. Und entgegen unserer wissenschaftlichen Erwartung fragt sie, welche Blume sie abschneiden solle. Vor ihr steht eine rote Rose, die aber nicht duftet und keinen dornigen Stiel hat und so rot ist wie Nelken, etc. Hat sie einen Fehler gemacht oder ist an unserer Definition etwas falsch? Danach ersuche ich sie, mir die Zierpflanze, die farblich in der Wellenlänge von $600 \mathrm{~mm}$ bis $780 \mathrm{~mm}$ liegt abzuschneiden, und sie hält mich für etwas sonderbar. Zwar versteht sie die Zahlen, aber sie kann sie hier nicht in einen Zusammenhang bringen, da es keine so grosse Anzahl von Blumen gibt und weil sie das Wort Wellenlänge zunächst nur mit den ihr bekannten Meereswellen in Verbindung bringt. Wenn ich sie weiters auffordern würde, mir eine Blume zu schenken, die von politisch links stehenden Parteien als Symbol benutzt wird, dann würde sie bestimmt irgendwelche Blumen abschneiden. Hat sie falsch gehandelt? Nein, wenn man ihr durch eine deiktische Handlung einen Hinweis geben würde, so könnte sie gemäss L. Wittgenstein antworten: "Du wolltest also eigentlich sagen..." kleine Tochter braucht die formenreichen Arten nicht $\mathrm{zu}$ kennen, um zwischen einer Rosa und einem Hibiscus rosa-sinensis unterscheiden zu können. Sie braucht auch nicht die physikalische Regel und die Theorie über die Wellenlängen oder parteitypische Symbole zu kennen, um die richtige Pflanze zu finden. Obwohl alle die hier aufgezählten Bestimmungen und

\footnotetext{
${ }^{11}$ Wittgenstein $B T, 2000,178$.

12 Ich werde hier nicht auf die Frage eingehen, ob diese Einsetzung durch die Identifizierung gemacht werden kann. Siehe: Padilla Gálvez 1989 und Padilla Gálvez 1988, 685-692.

${ }^{13}$ Wittgenstein $B T, 2000,178$.
} 
Beschreibungen in E. Husserls Vorträgen weder Unsinn noch widersinnige Ausdrücke waren, würden sie trotzdem zu keiner eindeutigen Definition von "Rose" führen. Unverträgliche grammatische Verknüpfungen sind seiner Auffassung nach nicht unbedingt sinnlos oder absurd. Was ein Hörer wissen muss ist, ob dessen Antwort (hier das Schneiden der richtigen Pflanzen) in unserem Spiel als Antwort auf meine Frage gilt. In unserem Spiel muss die Antwort auf die Frage folgen, aber so, dass beide miteinander in Beziehung stehen. Daher ist es relevant, das Spiel so zu analysieren, dass die Sprache des Spiels, bzw. dessen Grammatik, unter dem Gesichtspunkt eines Kalküls angesehen wird und als ein Operieren nach festgesetzten Regeln analysiert wird. ${ }^{14}$ Nur so können wir untersuchen, ob korrekt gespielt wird oder nicht.

\section{Die grammatische Methode}

In den 'Bemerkungen zur Philosophie' behauptet L. Wittgenstein "Philosophieren ist, /:/ falsche Argumente zurückzuweisen."15 Aufgrund dieser Sichtweise lässt sich die folgende Frage stellen: Hat es einen Sinn zu sagen, dass ich eine Rose in meinem Gesichtsraum sehe? Wir haben oben darauf hingewiesen, dass die Behauptung von L. Wittgenstein als unsinnig bestimmt wird. Nach dem Grund der Unsinnigkeit gefragt antwortet er:

"Wenn wir von Gesichtsraum reden, so werden wir leicht zu der Vorstellung verführt, als wäre er eine Art von Guckkasten, den jeder mit / vor / sich herumtrüge. D.h. wir verwenden dann das Wort "Raum" ähnlich, wie wenn wir ein Zimmer einen Raum nennen. In Wirklichkeit aber bezieht sich doch das Wort "Gesichtsraum" nur auf eine Geometrie, ich meine, auf einen Abschnitt der Grammatik unserer Sprache." ${ }^{\prime 6}$

In diesem Zitat nimmt er auf das Modell des "Guckkastens" Bezug, wie es J. Kepler ${ }^{17}$ vorschlug und S. Hoogstraten ${ }^{18}$ repräsentierte. Schon R. Descartes hatte diesem Modell folgendes entgegengesetzt:

\footnotetext{
${ }^{14}$ Wittgenstein $B T, 2000,180$.

${ }^{15}$ Wittgenstein $B P,(\mathrm{WA}, 4), 1999,12$.

${ }^{16}$ Wittgenstein $B T, 2000,310$.

${ }^{17}$ Kepler 1604, 56 ff.

18 van Hoogstraten, 1670. Ein solcher Guckkasten diente der Untersuchung der perspektivischen Gegebenheiten und Raumeinheiten mit den darin angeordneten Objekten.
} 
“... mais cela n'empesche pas qu'il ne foit vray que les objets que nous regardons, en impriment d'affés parfaites dans le fonds de nos yeux; ainsi que quelques vns ont desia tres-ingenieusement expliqué, par la comparaison de celles qui paroissent dans vne chambre, lors que l'ayant toute fermée, reserué vn seul trou, \& ayant mis au deuant de ce trou vn verre en forme de lentille, on estend derriere, a certaine distance, vn linge blanc, sur qui la lumiere, qui vient des objets de dehors, forme ces images." ${ }^{19}$

R. Descartes kritisiert die Darstellung des, gleichsam in dem überschaubaren Raum eines Guckkasten fungierenden unbewegten Auges. Der Guckkasten wurde zur Betrachtung von Bildern entwickelt, die durch Spiegel und Linsen scheinbar realistischer wirken. Der Guckkasten hat sich zu jenem Modell entwickelt, auf dem die subjektive Philosophie gründet. ${ }^{20}$ Die Betrachtung eines Bildes ist subjektlos. Wenn wir dennoch davon sprechen, dass ich im Guckkasten einen Berg sehe, so kann ich mit dem Personalpronomen nicht auf mich als eine Person unter anderen Bezug nehmen. Bilder haben also keine personalen Besitzer. Sieht man "Bilder" durch den Guckkasten, so kommt man darin nicht als personaler Besitzer vor. Sie sind bereits dann sinnvoll beschrieben, wenn man ihren "Ort" im Guckkasten bestimmt und sie so beschrieben werden können. Man hat von einer personalen Besitzerlosigkeit gesprochen, die auf eine innere Struktur hinweist. ${ }^{21}$ Diese personale Besitzerlosigkeit entspricht dem Vorgang, den man bei der Rückbezüglichkeit vollzieht. ${ }^{22}$

Wittgenstein kritisiert dieses Guckkasten-Modell, da das visuelle Bild innerhalb des Kastens auf Projektion beruht. Dieses Gleichnis zwischen Bild und Projektion ist innerhalb des szientistischen Denkens nie durchdacht worden, sondern wird immer vorausgesetzt, da wir das Verhältnis beider zueinander immer im Abbildmodell ansehen. Ein Sprecher sagt: "Ich sehe die Berge", aber niemals sagt er: "Ich sehe die Reproduktion der Berge in einer Abbildung", sondern eher: "Ich sehe die Abbildung der Berge". Der Betrachter müßte dann genau genommen sagen: "Ich sehe das reflektierte Bild der Berge (bzw. das reflektierte Bild der Bilder der Berge)," da im

${ }^{19}$ Descartes 1996, $114 \mathrm{f}$.

${ }^{20}$ Padilla Gálvez 2001, 259-273; Padilla Gálvez 2005, 53-79.

${ }^{21}$ Siehe: Lütterfelds 2006, 225.

${ }^{22}$ Siehe: Padilla Gálvez 1995, 1-25; Padilla Gálvez 1995, 97-104; Padilla Gálvez 2004, 137-153. 
Spiegel die Berge und deren Abbild nicht eindeutig unterschieden werden können. Im Spiegel werden sowohl die Berge, als auch deren Abbild zweidimensional wiedergegeben. Das Fehlen einer solchen Explizitheit macht die Beschreibung so konfus. Die eindeutige Bestimmung des Abschnitts über die Grammatik der Sprache erlaubt uns, ihre Inkongruenzen zu erkennen. Die Sekundärliteratur analysiert Wittgensteins Grammatik-Begriff aus verschiedenen Blickwinckeln. Für Rosaria Egidi wird Grammatik auf eine "phänomenologische Aufgabe" reduziert. ${ }^{23}$ Für Christian Stetter steht die "Schulbedeutung des Wortes "Grammatik", des Kompendiums von Regeln noch im Wege." ${ }^{24}$ Sabine Knabenschuh de Porta vertritt die Auffassung, dass die grammatikalische Einstellung Wittgensteins eine Art "vitaler Holismus" sei. $^{25}$

Im Zitat wird eindeutig darauf hingewiesen, dass das Wort "Gesichtsraum" nur auf eine Geometrie Bezug nimmt und daher nur einen "Abschnitt" der Grammatik darstellt. Die Frage besteht folglich darin, zu wissen, welcher Abschnitt hier gemeint sein kann. E. Husserl hat in den Logischen Untersuchungen darauf hingewiesen, dass er die apriorische Grammatik aufrecht erhalten wollte. Diese Grammatik sollte als “... die mögliche Bedeutungsformen bestimmender Gesetze ein zweifelloses Fundament" erhalten. ${ }^{26}$ Nach seiner Auffassung gehören die Gesetze zur Konstitution der wesentlichen Bedeutungsformen. Die Verträglichkeit bzw. Unverträglichkeit der Bedeutung wird bedingt durch die "...Möglichkeit oder Unmöglichkeit des Seins von bedeuteten Gegenständen (...), sofern sie durch das eigene Wesen der Bedeutungen bedingt und somit aus diesem in apodiktischer Evidenz einsehbar ist." 27 Der Gegensatz zwischen Objektivität und Unsinnigkeit wird - wie wir oben rekonstruiert haben - zwar vorausgesetzt, aber nicht erklärt.

L. Wittgenstein lehnt die Husserlsche Auffassung von Grammatik ab. Bevor wir uns hier mit seiner eigenen Position auseinandersetzen, wollen wir seine Zugangsweise analysieren. Er widmet sich der Frage, ob die Gesichtsräume einen Besitzer haben. In dieser Hinsicht ist er sehr explizit,

${ }^{23}$ Egidi 1989, 199.

${ }^{24}$ Stetter 2006, 114.

${ }^{25}$ Knabenschuh de Porta 2007, 90.

${ }^{26}$ Husserl $L U, \mathrm{II} / 1,1968,295$.

${ }^{27}$ Husserl $L U$, II/1, 1968, 334. 
denn er entgegnet, dass Gesichtsräume weder einen "Besitzer" haben, noch dass sie jemandem gehören würden. Letzteres benennt er mit einem lateinischen Ausdruck als "vazierende" Gesichtsräume, was eine veraltete Ausdrucksweise ist und "frei von Gesichtsraum" als auch "unbesetzt" meint. ${ }^{28}$ Die von L. Wittgenstein angewendete Argumentationsfigur kann folgendermaßen zusammengefasst werden. Wenn sich der Terminus "Gesichtsraum" allein auf eine Geometrie bezieht, so ist er objektiv. Daher ist ein Gesichtsraum ohne Besitzer und ihm fehlt jegliche Subjektivität. Damit ist der Gesichtsraum innerhalb eines objektiven Schnitts zu analysieren. Der Gesichtsraum muss als Abschnitt der Grammatik der geometrischen Sprache untersucht werden. Diese Geometrie wird aber als ein Abschnitt der Grammatik unserer Sprache bestimmt und ihre Bestimmungen werden wie folgt zusammengefasst:

"Das Wesentliche der Töne und Farben ist offenbar in der Grammatik der Wörter für die Töne und Farben gezeigt.,"29

Folglich müssten wir die Grammatik bestimmen, die man beim Gebrauch der Wörter "Töne" und "Farben" anwendet. Grammatik und Wirklichkeit werden als getrennte Bereiche angesehen, die sich nicht gegenseitig bedingen. Sie stehen nicht in Rechtfertigungszusammenhang. Auf die Wörter "Töne" und "Farben" wird eine Grammatik angewendet, die ganz im Gegensatz zu Husserls Bestimmung - nicht für die Realität haftet. So sagt Wittgenstein:

"Die Grammatik ist keiner Wirklichkeit verantwortlich.

(Die Grammatik ist der Wirklichkeit nicht Rechenschaft schuldig.)" $" 30$

Durch dieses Zitat spricht L. Wittgenstein mehrere Probleme an: Wenn von der Grammatik kein Bezug zur Realität verlangt wird, wie entsteht dann eine Korrelation zwischen beiden Bereichen? Wieder ist L. Wittgenstein sehr explizit, wenn er als einziges Korrelat zwischen der Sprache und der Wirklichkeit die Willkürlichkeit der grammatischen Regeln stellt. ${ }^{31} \mathrm{Er}$ bezeichnet die Regeln der Grammatik als "willkürlich", weil sie nicht durch ihren Wirklichkeitsbezug definiert werden. Wenn ein Sprecher andere

\footnotetext{
${ }^{28}$ Wittgenstein $B T, 2000,310$.

${ }^{29}$ Wittgenstein $B T, 2000,310$.

${ }^{30}$ Wittgenstein $B T, 2000,165$.

${ }^{31}$ Wittgenstein $B T, 2000,166$.
} 
grammatische Regeln als die üblichen gebraucht, muss er nicht unbedingt etwas Falsches aussprechen, er könnte auch einfach auf etwas anderes Bezug genommen haben. Nehmen wir an, ich soll den deutschen Satz "die Rose wächst rechts in meinem Garten" im Spanischen ausdrücken, durch:

La rosa en mi jardín crece a la derecha.

Obwohl der Satz in (3) eine andere Grammatik aufweist als die deutsche, so heisst es nicht unbedingt, dass der Satz (3) etwas anderes aussagt. Ein deutscher Hörer, der der spanischen Sprache nicht kundig ist, könnte bezweifeln, dass ein in einer Sprache durch sieben Wörter ausgedrückter Sachverhalt diesselbe Bedeutung habe wie das, was in seiner Sprache durch neun Wörter zum Ausdruck gebracht wird. In diesem Zusammenhang betont Wittgenstein, dass eine Sprache das ist, was sie ist "... und eine andere Sprache ist nicht diese Sprache." 32 Somit können sich zwei verschiedene Sprachen nicht gegenseitig bestimmen, sondern behalten vom grammatikalischen Standpunkt aus gesehen ihre Unabhängigkeit. Die Ausdrucksfähigkeit des Deutschen kann von der spanischen Sprache unterschieden sein durch die Anzahl der Wörter, die man gebraucht, obwohl die grammatikalischen Regeln entsprechend verwendet werden.

\section{Wider den Konventionalismus}

Mit dem oben genannten Argument entscheidet sich L. Wittgenstein gegen den Konventionalismus. Er sagt andernorts: "Ich nenne Regeln der Darstellung nicht Konventionen, wenn sie sich dadurch rechtfertigen lassen, daß die Darstellung, wenn sie ihnen gemäß ist, mit der Wirklichkeit übereinstimmt." ${ }^{, 3}$ Es geht darum, herauszufinden, ob sich die Konventionen der Grammatik durch die Beschreibung des Dargestellten begründen lassen. ${ }^{34}$ Der Konventionalist behauptet, dass der Sprecher in immer wiederkehrenden Situationen eine bestimmte Regel verwendet. Wenn nun eine ähnliche Situation auftritt, dann müsste der Sprecher dieselbe Regel verwenden. Von jedem anderen Sprecher wird ebenfalls erwartet, dass er in ähnlichen Situationen dieselbe Regel befolgt. Der Konventionalist geht davon aus, dass

\footnotetext{
${ }^{32}$ Wittgenstein $B T, 2000,165$.

${ }^{33}$ Wittgenstein $P G, 1969,186$.

${ }^{34}$ Vgl. Wittgenstein $B T, 2000,168$.
} 
eine grammatikalische Regel zum gemeinsamen Wissen gehört, wenn jeder Sprecher einer Sprachgemeinschaft die Regel befolgt, nahezu jeder von nahezu jedem anderen erwartet, dass der Sprecher die Regel befolgt. Dabei wird immer vorausgesetzt, dass jeder Sprecher dieselben sprachlichen Präferenzen habe und jeder zu einer Sprachgemeinschaft Zugehörige die Regeln befolgt.

L. Wittgenstein lehnt diese Position ab. Im Falle von Farbausdrücken ist die Aussagekraft des Konventionalismus aus zweierlei Gründen eingeschränkt und daher abzulehnen. Erstens, wenn ein Sprecher die grammatischen Konventionen in der Weise beschreiben würde, dass man der Farbe gewisse Eigenschaften zuschriebe, so würde das die Konvention selbst unnötig machen. In diesem Fall könnte nämlich jeder Sprecher das aussprechen, was die Konvention ausschließen würde. Wenn, zweitens, die Konventionen als Kriterium für den Ausschluss bestimmter Wortkombinationen benutzt würden, so könnte man Farben nicht bestimmte Eigenschaften zuschreiben. Dafür würde man nämlich Konventionen brauchen. Wenn die Farben diese Eigenschaften hätten, dann würde das wiederum gegen die Konventionen verstoßen. Man muss sich in Erinnerung rufen, dass die Beschreibung des Dargestellten immer Grammatikregeln voraussetzt. Daher ist der Konventionalismus keine Lösung des Problems, sondern hindert uns eher daran, zu erkennen, wo die eigentlichen Probleme liegen.

Wenn die Regeln nicht konventionell bestimmbar sind, so stellt sich die Frage, was Regeln eigentlich sind. Was versteht L. Wittgenstein genau unter einer "Regel"? Er antwortet mit folgender Metapher:

"Die Regel - wie ich sie verstehe - ist wie ein Weg in einem Garten., 35

Das Zitat scheint prima facie alles andere als eindeutig $\mathrm{zu}$ sein. Offensichtlich wird die Metapher deswegen verwendet, weil der Begriff der "Regel" selbst undeutlich ist. Der Begriff "Regel" ist nach Wittgenstein selbst verschwommen und unscharf.

“"Regel' ist in dem Sinne ein Begriff mit verschwommenen Rändern, wie 'Blatt' oder' 'Stiel' oder 'Tisch', etc.." 36

${ }^{35}$ Wittgenstein $B T, 2000,171$.

${ }^{36}$ Wittgenstein $P G,(\mathrm{WA}, 5), 1996,30$. 
Bei der Untersuchung der strikten Grammatik der Regeln stößt L. Wittgenstein immer wieder auf das Problem der unscharfen Grenzen der Begriffe. Er betont, dass viele Begriffe nicht scharf begrenzt werden können. Was versteht er aber in diesem Zusammenhang unter der Metapher "Weg in einem Garten"? Ein Weg ist normalerweise ein begehbarer Streifen in einem wenig ausgebauten Gelände, der einem die Erreichbarkeit eines bestimmten Bereiches erleichtert. Dieser Weg soll aber nicht als Mittel für einen bestimmten Zweck erforscht werden.

Diese metaphorische Bestimmung der Regel ist ganz anders als die übliche Regelbestimmung, die als eine begrifflich bestimmte Gleichförmigkeit oder Konstanz definiert wird. I. Kant stellte den Begriff der "Regel" a priori auf, in welcher eine Vereinigung von Vorstellungen als notwendig gedacht wird. Somit definiert er die Regel als "Urteile, sofern sie bloß als die Bedingung der Vereinigung gegebener Vorstellungen in einem Bewußtsein betrachtet werden." 37 L. Wittgenstein wendet sich von einer solchen Definition ab.

Er weist auf zwei Momente hin, die den Begriff der Regel bestimmen, zum einen sieht er in der Verwechslung zwischen physikalischer und phänomenologischer Sprache den Ursprung einer Paradoxie. Wenn man versucht, die Verschwommenheit der Begriffe durch unklare Begriffe wiederzugeben, so redupliziert man das Problem. Somit kann man die unklare Bestimmung des Begriffs der Regel nicht eindeutig machen. Damit hängt das zweite Moment zusammen, dass nämlich die Undeutlichkeit eine "interne Eigenschaft" ${ }^{\prime 3}$ sei. Diese interne Eigenschaft ist beispielsweise dafür verantwortlich, dass wir die Grenzenlosigkeit des Gesichtsraumes konzipieren. Die oben genannte Argumentation wird in den Philosophischen Untersuchungen noch klarer herausgearbeitet, wo der Begriff "Regel" durch "Spiel" ersetzt und wie folgt dargestellt wird:

"Man kann sagen, der Begriff 'Spiel' ist ein Begriff mit verschwommenen Rändern." 39

Wittgenstein fragt sich daraufhin, ob ein verschwommener Begriff überhaupt ein Begriff sei. Im Gegensatz zu G. Frege, der mit "unbegrenzten

\footnotetext{
${ }^{37}$ Kant 1983, § 23, 172.

${ }^{38}$ Wittgenstein $B T, 2000,313$.

${ }^{39}$ Wittgenstein $P U, 2001, \S 71$.
} 
Bezirken" nichts anfangen konnte, versucht Wittgenstein eine minimalistische Theorie der Verschwommenheit zu entwickeln. Er lehnt selbst die Möglichkeit ab, Unschärfe durch Schärfe zu ersetzen. ${ }^{40}$

Selbstverständlich ist ein Weg in einem Garten alles andere als eine "Regel a priori", da sich die Funktion eines Weges in der Erreichbarkeit eines bestimmten Bereiches erschöpft. Er ist eine Art "vorgezeichnetes Feld". Somit wird der Weg - in der Regel - “... nur als Instrument der Darstellung verstanden werden." festgelegten Regeln analysiert. Gemäß dem von E. Husserl vorgeschlagenen Beispiel stellt L. Wittgenstein fest, dass der Gebrauch der Wörter "rund" und "Viereck" dann als sinnlos anzusehen ist, wenn sich am selben Ort und zur selben Zeit ein Kreis in einem Viereck befindet. Die Sinnlosigkeit eines solchen Satzes liegt in seiner Kontradiktion. Dieser Satz sagt nichts über das Objekt aus, das wir uns vorstellen sollen und das gleichzeitig rund und viereckig sein sollte. Um den Unterschied noch besser hervorzuheben, muss man die Regel in ihrer Beziehung zu den Sätzen stärker hervorheben. In dieser Hinsicht verhält sich eine Regel zu einem Erfahrungssatz ähnlich wie die Zeichnung eines Wohnhauses zu dessen Beschreibung. So sagt L. Wittgenstein:

"Eine Regel verhält sich zu einem Erfahrungssatz ähnlich, wie die Zeichnung, die die charakteristischen Merkmale eines Wohnhausplanes hat, zu der Beschreibung, welche sich einer solchen Zeichnung bedient, und welche sagt, daß so ein Haus dort und dort existiere / stehe/." ${ }^{42}$

Daraus ergibt sich seine Absicht, ein Regelverzeichnis unserer Sprache aufzustellen, um strikte grammatische Regeln zur Verwendungsweise der Begriffe zu kennzeichnen. Bei diesem Unternehmen stößt er auf einen relevanten Aspekt, nämlich die sogenannte "unscharfe Grenze" der Begriffe. ${ }^{43}$

\footnotetext{
${ }^{40}$ Wittgenstein $P U, 2001, \S 77$.

${ }^{41}$ Wittgenstein $B T, 2000,172$.

${ }^{42}$ Wittgenstein $B T, 2000,173$.

43 Der Begriff "Regel" ist in 'The Big Typescript' nach Wittgenstein selbst verschwommen und unscharf. "Regel' ist in dem Sinne ein Begriff mit verschwommenen Rändern, wie 'Blatt' oder' 'Stiel' oder 'Tisch', etc." (Wittgenstein $P G, 1996,30$ ). Um dies noch schärfer klarzustellen müssen wir einen kurzen Hinweis auf die späteren Werke machen, wo das Thema eindeutiger dargestellt wird als im Jahr 1933. Die oben genannte Argumentation wird in den Philosophischen Untersuchungen klarer herausgearbeitet, wo
} 


\section{Schluß}

L. Wittgenstein hat in Zusammenhang mit den Problemen um das "runde Viereck" auf die Diskussion zwischen Sokrates und Theaitetos Bezug genommen. Dort findet sich folgender Dialog:

"Sokrates: Wer also vorstellt, was nicht ist, der stellt nichts vor?

Theaitetos: So scheint es.

S.: Wer aber nichts vorstellt, der wird gewiß überhaupt gar nicht vorstellen?

Th.: Offenbar, wie wir sehen." 44

L. Wittgenstein schlägt dazu folgendes Gedankenexperiment vor:

"Setzen wir in diesem Argument |[und dem |ihm| vorherstehenden]| statt "vorstellen" etwa "zerschneiden" / "töten" / so läuft es auf eine Regel der Verwendung dieses Wortes hinaus. Man dürfe nicht sagen / Es hat keinen Sinn zu sagen /: "ich zerschneide /töte/ etwas was nicht existiert.".,45

L. Wittgenstein betont, dass der Unterschied zwischen den Begriffen "Vorstellung" und "Zerschneiden" nicht auf irgendwelche gegenständlichen Elemente zurückgeführt werden kann, sondern eher in der Regel ihrer Verwendungsweise liegt. Folglich kann man sagen, dass der Unterschied zwischen der Bedeutung beider Wörter von der Grammatik abhängig ist.

In der Welt Platons kann man sich kein "rundes Quadrat" vorstellen, das gleichzeitig rund und quadratisch wäre. Wenn man sich ein nicht existierendes, rundes Quadrat vorstellen will, bedeutet das, dass man sich ein rundes Quadrat vorstellt, obwohl keines da ist. Die Diagonale eines runden Quadrats zu zerschneiden hieße aber nicht, dass ein rundes Quadrat da sein müsste und dadurch verschiedene grammatische Regeln garantiert sind. Wenn Sokrates behaupten würde, um mir ein rundes Quadrat vorzustellen, muss es dieses doch in einem gewissen Sinne geben, so verneint Wittgenstein das

der Begriff "Regel" durch "Spiel” ersetzt und wie folgt dargestellt wird: "Man kann sagen, der Begriff 'Spiel' ist ein Begriff mit verschwommenen Rändern. Aber ist ein verschwommener Begriff überhaupt ein Begriff? [...] Frege vergleicht den Begriff mit einem Bezirk und sagt: einen unklar begrenzten Bezirk könne man überhaupt keinen Bezirk nennen. Das heißt wohl, wir können mit ihm nichts anfangen." (Wittgenstein $P U$, 2001, § 71).

${ }^{44}$ Wittgenstein BP, (WA, 4), 1999, 12. Vgl.: Platon 1940, 629.

${ }^{45}$ Wittgenstein $B P,(\mathrm{WA}, 4), 1999,12$. 
eindeutig. Würde Sokrates hingegen argumentieren, dass die Existenz der roten Farbe eine Voraussetzung dafür ist, dass man sie sich vorstellen kann, so wäre L. Wittgensteins Antwort, dass dessen Existenz nur als Färbung eines Gegenstandes angesehen werden kann. In 'The Big Typescript' bricht Wittgenstein mit jeder Eindeutigkeit und entdeckt die Verschwommenheit der Begriffe, die das Problem des Sehens beeinträchtigen.

\section{Literatur}

Descartes, René, 1996: La Dioptrique. In: CEuvres de Descartes, (Publiées par Charles Adam \& Paul Tannery), Bd. VI, J. Vrin, Paris, 79-228.

Egidi, Rosaria, 1989: Phänomenologie, Psychologie und Grammatik in Wittgenstein, Wittgenstein im Focus - Im Brennpunkt: Wittgenstein, (Hrsg. von Brian McGinnes und Rudolf Haller), Rodopi, Amsterdam, 185-205.

Hoogstraten, Samuel van, 1670: Inleyden tot de Hooge Schoole der Schilderkunst: Anders de Zichbare Werelt, Rotterdam.

Husserl, Edmund, 1968: Logische Untersuchungen. Untersuchungen zur Phänomenologie und Theorie der Erkenntnis, II/1, Max Niemeyer, Tübingen.

Kant, Immanuel, 1983: Prolegomena zu einer jeden künftigen Metaphysik, die als Wissenschaft wird auftreten können, in: I. Kant, Werke in sechs Bände (Hrsg. von W. Weischedel), Bd. III, Wissenschaftliche Buchgesellschaft, Darmstadt.

Kepler, Johann, 1604: Ad Vitellionem paralipomena, quibus astronomice pars optica traditur; Potissimùm de artificiosa observatione et astimatione diametrorum deliquiorumq; Solis \& Lunce.[...], Claudium Marnium \& Hæredes Ioannis Aubrii, Frankfurt.

Knabenschuh de Porta, Sabine, 2007: Gramática como principio experiencial: El holismo vital de Wittgenstein, in: El laberinto del lenguaje (Hrsg. Jesús Padilla Gálvez), Ediciones UCLM, Cuenca, 75-94.

Lütterfelds, Wilhelm, 2006: "Schmerz haben". Wittgensteins frühes Plädoyer für eine solipsistische Privatheit des Innere, in: Stefan Majetschak (Hrsg.), Wittgensteins 'große Maschinenschrift', Peter Lang, Frankfurt a.M.; Berlin; Bern; Bruxelles; New York; Oxford; Wien, 219-246.

Mach, Ernst, 1922: Analyse der Empfindungen und das Verhältnis des Physischen zum Psychischen, Verlag von Gustav Fischer, Jena. (Nachdruck der Wissenschaftlichen Buchgesellschaft, Darmstadt, 1991).

Meinong, Alexius, 1978: Über Gegenstandstheorie, in: A. Meinong, Gesamtausgabe. Bd. II, Akad. Druck- und Verlagsanstalt, Graz, 481-530. 
Padilla Gálvez, Jesús, 1988: Identität und Selbigkeit. Ontologische Interpretation der Leibnizschen Individuen, in: V. Internationaler Leibniz-Kongreß. Leibniz Tradition und Aktualität (Hrsg. A. Heinekamp), Hannover, 685-692.

Padilla Gálvez, Jesús, 1989: Referenz und Theorie der möglichen Welten. Darstellung und Kritik der logisch-semantischen Theorie in der Sprachanalytischen Philosophie, Peter Lang, Frankfurt a. M.

Padilla Gálvez, Jesús, 1995: Die Verwendung des Wortes "Ich" bei L. Wittgenstein, Wittgenstein Studies, 3, 1-25.

Padilla Gálvez, Jesús, 1995: Gibt es in der Sprache ein metaphysisches Subjekt? Culture and Value. Philosophy and the Cultural Sciences, Kirchberg am Wechsel, 97-104.

Padilla Gálvez, Jesús, 2001: Nichts ist dunkler als das Licht. Das Problem der Farben bei Wittgenstein, Goethe, Rizzetti und Newton, Philosophisches Jahrbuch, 108 /II, 259-273.

Padilla Gálvez, Jesús, 2004: Disgressionen über das sich entwerfende Ich. In: Die Wiederkehr des Idealismus? Festschrift für Wilhelm Lütterfelds zum 60. Geburtstag. Hrsg. von Thomas Mohrs, Andreas Roser und Djavid Salehi, Peter Lang, Frankfurt a.M.; Berlin; Bern; New York; Oxford; Wien, 137-153.

Padilla Gálvez, Jesús, 2005: Minima visibilia, Episteme NS, 25, 53-79.

Platon, 1940: Theaitetos, in: Sämtliche Werke, Bd. 2, Lambert Schneider, Berlin, 561662.

Russell, Bertrand, 1956: On Denoting, in: B. Russell, Logic and Knowledge. Essays 19011950, (Ed. by R. Ch. Mersh), George Allen \& Unwin, London, 1956, 41-56.

Stetter, Christian, 2006: Der Begriff der Grammatik in Wittgensteins Big Typescript, in: Wittgensteins 'große Maschinenschrift', (Hrsg. Stefan Majetschak), Peter Lang, Frankfurt a. M., 99-114.

Wittgenstein, Ludwig, 1969: Philosophische Grammatik, in: Schriften, Bd. 4, Suhrkamp, Frankfurt a. M.

Wittgenstein, Ludwig, 1996: Philosophische Grammatik, in: Wiener Ausgabe, (WA, 5), (Hrsg. M. Nedo), Springer, Wien - New York.

Wittgenstein, Ludwig, 1999: Bemerkungen zur Philosophie, in: Wiener Ausgabe, (WA, 4), (Hrsg. M. Nedo), Springer, Wien - New York.

Wittgenstein, Ludwig, 2000: The Big Typescript, in: Wiener Ausgabe, (WA, 11), (Hrsg. M. Nedo), Springer, Wien - New York.

Wittgenstein, Ludwig, 2001: Philosophische Untersuchungen. Kritisch-genetische Edition, (Hrsg. Joachim Schulte), Suhrkamp, Frankfurt a. M. 